\title{
Ultrahigh-Energy Photons as a Probe of Nearby Transient Ultrahigh-Energy Cosmic-Ray Sources and Possible Lorentz-Invariance Violation
}

\author{
Kohta Murase \\ Yukawa Institute for Theoretical Physics, Kyoto University, Kyoto 606-8502, Japan
}

(Dated: July 29)

\begin{abstract}
Detecting neutrinos and photons is crucial to identifying the sources of ultrahigh-energy cosmic rays (UHECRs), especially for transient sources. We focus on ultrahigh-energy $\gamma$-ray emission from transient sources such as $\gamma$-ray bursts, since $>\mathrm{EeV} \gamma$ rays can be more direct evidence of UHECRs than $\sim \mathrm{PeV}$ neutrinos and $\mathrm{GeV}-\mathrm{TeV} \gamma$ rays. We demonstrate that coincident detections of $\sim 1-100$ events can be expected by current and future UHECR detectors such as Auger and JEM-EUSO, and the detection probability can be higher than that of neutrinos for nearby transient sources at $\lesssim 50-100 \mathrm{Mpc}$. They may be useful for constraining the uncertain cosmic radio background as well as knowing the source properties and maximum energy of UHECRs. They can also give us more than $10^{4}$ times stronger limits on the Lorentz-invariance violation than current constraints.
\end{abstract}

PACS numbers: 98.70.Rz, 11.30.Cp, 98.70.Sa

The origin of ultrahigh-energy cosmic rays (UHECRs) is one of the biggest mysteries in astroparticle physics, and a number of scenarios have been theoretically proposed so far (for reviews, see, e.g., [1]). However, physical conditions in these potential sources are uncertain, and observational progress in source identification has been limited by the scarcity of experimental data (e.g., 2]). The recent results of large area detectors such as the Pierre Auger Southern Observatory (PAO) have started to give us crucial clues to the origin. Indeed, the first PAO results reported a significant correlation between the arrival directions of the highest-energy cosmic rays and the large-scale structure of the Universe, which is inhomogeneous up to dozens of Mpc (e.g., 3, 4n). However, not only active galactic nuclei (AGNs) [5, 6] but also transient sources such as $\gamma$-ray bursts (GRBs) [7, 8] and magnetars [9] can be UHECR sources so far. Even if the association of UHECRs with AGNs is real, the PAO report suggests that the majority of the correlating AGNs seems radio-quiet, a class of objects not showing any nonthermal high-energy emission, and the power of those AGNs seems insufficient to produce UHECRs [10]. This problem may be solved if UHECRs are produced during active states such as flares [11, 12]. When the UHECR sources are transient, the magnetic fields in the Universe not only deflect UHECRs but also cause significant time delays compared to photons and neutrinos generated during the bursts (e.g., [13]). Then, due to difficulties in identifying the sources through UHECRs, it is more favorable to detect photons and neutrinos.

We focus on ultrahigh-energy (UHE) photon emission from transient UHECR sources with numerical calculations considering the cosmic infrared, microwave, or radio background ( $\mathrm{CIB} / \mathrm{CMB} / \mathrm{CRB}$ ) and the loss due to the intergalactic magnetic field (IGMF). We demonstrate that UHE photons can be the most useful messenger for nearby sources, though the results depend on source properties and the uncertain CRB. Constraints on the Lorentz-invariance violation (LIV) are also discussed.

UHE photon production in the source. - If cosmic rays are accelerated up to ultrahigh energies, hadronic $\gamma$ rays and neutrinos should be produced via the $p p$ or $p \gamma$ reactions, but their efficiency and resulting spectra depend on source models [8, 12, 14, 15]. In this work, for demonstrative purposes, we mainly consider $p \gamma$ photons and neutrinos from GRBs [16] as an example (e.g., [8, 14, 15]). We especially demonstrate the case of relatively low luminous bursts, motivated by recent suggestions that nearby bursts such as GRB 060218 are dimmer but more numerous than classical GRBs [and they are often called lowluminosity (LL) GRBs] [17]. Other cases such as AGN flares can also be considered similarly.

First, we write a source UHECR energy spectrum as

$\tilde{\mathcal{E}}_{\mathrm{CR}}^{\text {iso }} \equiv E_{p}^{2} \frac{d N_{p}^{\text {iso }}}{d E_{p}} \approx \frac{1}{\rho}\left(E_{p}^{2} \frac{d \dot{N}_{\mathrm{CR}}}{d E_{p}}\right)_{E_{p}=E_{0}}\left(\frac{E_{p}}{E_{0}}\right)^{2-p} \mathrm{e}^{-E_{p} / E_{p}^{\max }}$,

where $\rho$ is the local apparent rate of bursts responsible for the observed UHECRs, $p$ is the source spectral index, and $E_{p}^{\max }$ is the maximum UHECR energy. In this work, assuming proton composition, we adopt $p=2$ expected in the ankle scenario [1, 2]. The energy input rate at $E_{0}=10^{19} \mathrm{eV}$ is estimated as $E_{p}^{2} \frac{d \dot{N}_{C R}}{d E_{p}} \sim$ $10^{44} \mathrm{ergMpc}^{-3} \mathrm{yr}^{-1}$ from the UHECR data [1, 7, 13. The recent PAO results suggest that, if the UHECR sources are transient, the UHECR energy input per burst at $10^{19} \mathrm{eV}$ is $\tilde{\mathcal{E}}_{\mathrm{HECR}}^{\text {iso }} \equiv \tilde{\mathcal{E}}_{\mathrm{CR}}^{\text {iso }}\left(10^{19} \mathrm{eV}\right) \sim 10^{50.5} \mathrm{erg} \rho_{2.5}^{-1}$ $\left(0.1 \mathrm{Gpc}^{-3} \mathrm{yr}^{-1} \lesssim \rho \lesssim 10^{3.5} \mathrm{Gpc}^{-3} \mathrm{yr}^{-1}\right)$ [13]. Classical GRBs correspond to $\rho \sim 0.1-1 \mathrm{Gpc}^{-3} \mathrm{yr}^{-1}$ while LL GRBs, hypernovae [17] and AGN flares [11] may correspond to $\rho \sim 10^{2-3} \mathrm{Gpc}^{-3} \mathrm{yr}^{-1}$.

Provided a proton spectrum and a target photon spectrum, we can calculate spectra of $p \gamma$ photons and neutrinos. As a photon spectrum, we use a (broken) power law which is also expected in the synchrotron emission mechanism: $d n / d \varepsilon \propto \varepsilon^{-\alpha}$. Here $\varepsilon$ is the target photon energy in the comoving frame (while $\varepsilon_{\mathrm{ob}} \approx \Gamma \varepsilon$ is the energy in the observer frame, where $\Gamma$ is the bulk Lorentz factor). In the case of GRB prompt emission, 
$\alpha \sim 1$ for $\varepsilon<\varepsilon^{b}$ and $\alpha \sim 2$ for $\varepsilon^{b}<\varepsilon$ are observed as typical values, where $\varepsilon^{b}$ is the break energy [16]. Then, using the $\Delta$-resonance approximation, the effective optical depth for the $p \gamma$ reaction in the source is estimated as [8, 14] $f_{p \gamma} \approx t_{\mathrm{dyn}} / t_{p \gamma} \sim 0.1 \frac{L_{\gamma, 48}^{b}}{r_{14.5} \Gamma_{1.5}^{2} \varepsilon_{\mathrm{ob}, 10 \mathrm{keV}}^{b}}\left(E_{p} / E_{p}^{b}\right)^{\alpha-1}$, where $E_{p}^{b} \approx 1.6 \times 10^{16} \mathrm{eV} \Gamma_{1.5}^{2}\left(\varepsilon_{\mathrm{ob}, 10 \mathrm{keV}}^{b}\right)^{-1}$ is the resonance energy, $L_{\gamma}^{b}$ is the photon luminosity at $\varepsilon_{\mathrm{ob}}^{b}, r$ is the emission radius, $t_{\mathrm{dyn}} \approx r / \Gamma c$ is the dynamical time scale of the relativistic source, and $t_{p \gamma}$ is the $p \gamma$ energy loss time scale. We may expect efficient meson production $\left(\min \left[1, f_{p \gamma}\right] \sim 0.01-1\right)$.

The produced pions decay into $\gamma$ rays and neutrinos via $\pi^{0} \rightarrow 2 \gamma$ and $\pi^{ \pm} \rightarrow e^{ \pm}+\nu_{e}\left(\bar{\nu}_{e}\right)+\nu_{\mu}+\bar{\nu}_{\mu}$. Lifetimes of $\pi^{0}$ and $\pi^{ \pm}$are $8.4 \times 10^{-17}$ and $2.6 \times 10^{-8} \mathrm{~s}$, respectively. Because of $\pi^{0}$ 's very short lifetime, we may expect that sufficiently high-energy $\gamma$ rays reflect proton and photon spectra, leading to $E_{\gamma}^{2} \phi_{\gamma}^{\text {pri }} \propto f_{p \gamma} E_{\gamma}^{2-p} \propto E_{\gamma}^{1+\alpha-p}$. However, for $\gamma$ rays, it is an important issue whether they can escape from the source without significant source attenuation. The most relevant process is pair creation, whose optical depth is evaluated for the same photon field as that given for the $p \gamma$ reaction [8, 14] (see also Ref. [18] for more general discussions). But, for synchrotron sources, the self-absorption becomes important at low energies [19, 20]. In the case of GRB prompt emission, the synchrotron self-absorption energy is roughly estimated as $\varepsilon_{\mathrm{ob}}^{\mathrm{sa}} \sim 2 \mathrm{eV}\left(L_{\gamma, 48}^{b}\right)^{1 / 3} L_{M, 49}^{1 / 3} \xi_{B}^{1 / 3} \Gamma_{1.5}^{-2 / 3} r_{14.5}^{-1}$, where $L_{M}$ is the outflow luminosity and $\xi_{B}$ is the ratio of the magnetic energy density to the photon energy density [16, 19]. When the Klein-Nishina effect is relevant above $\tilde{\varepsilon}_{\mathrm{ob}}^{s a}\left(\right.$ where $\left.\tilde{\varepsilon}_{\mathrm{ob}} \equiv \Gamma^{2} m_{e}^{2} c^{4} / \varepsilon_{\mathrm{ob}}\right)$, we have [8, 14, 19, 20]

$\tau_{\gamma \gamma} \simeq 50\left(\frac{f_{p \gamma}\left(E_{p}^{b}\right)}{0.1}\right) \begin{cases}\left(\frac{E_{\gamma}}{\tilde{\varepsilon}_{\mathrm{b}}^{b}}\right)^{\alpha-1} & \left(E_{\gamma} \leq \tilde{\varepsilon}_{\mathrm{ob}}^{s a}\right) \\ \left(\frac{\tilde{\varepsilon}_{\mathrm{ob}}^{s a}}{\tilde{\varepsilon}_{\mathrm{ob}}^{b}}\right)^{\alpha-1}\left(\frac{E_{\gamma}}{\tilde{\varepsilon}_{\mathrm{ob}}^{s a}}\right)^{-1} \Lambda & \left(\tilde{\varepsilon}_{\mathrm{ob}}^{s a}<E_{\gamma}\right)\end{cases}$

where $\Lambda$ is the logarithmically energy-dependent term from the Klein-Nishina effect. An example for a somewhat bright LL GRB-like burst is shown in Fig. 1, where accurate cross sections of $\gamma \gamma \rightarrow$ $e^{+} e^{-}$and $\gamma e^{-} \rightarrow e^{-} e^{+} e^{-}$are used. Although the escapability depends on source models, UHE photons could escape from the source at $E_{\gamma}^{\text {thin }} \sim$ $10^{16} \mathrm{eV} \quad L_{\gamma, 48}^{b} r_{14.5}^{-1}\left(\varepsilon_{\mathrm{ob}, 10 \mathrm{keV}}^{b} \varepsilon_{\mathrm{ob}, 1 \mathrm{eV}}^{s a}\right)^{-1}\left(\tilde{\varepsilon}_{\mathrm{ob}}^{s a} / \tilde{\varepsilon}_{\mathrm{ob}}^{b}\right)^{\alpha-1} \Lambda$ unless additional low-energy photon fields exist. In this work, we calculate primary $\gamma$-ray spectra by exploiting elaborate numerical calculations including various processes [8, 15] and the result for the somewhat bright LL GRB-like burst is shown in Fig. 2, where $\xi_{B}=1$ and the other relevant parameters are described in the caption of Fig. 1. In the calculations, we also estimate the maximum energy and $E_{p}^{\max } \simeq 10^{20.5} \mathrm{eV}$ is obtained in this case. Roughly speaking, the primary $\gamma$-ray spectrum can be approximated as $E_{\gamma}^{2} \phi_{\gamma}^{\text {pri }} \approx$ $\frac{1}{4 \pi D^{2}} \frac{1}{2} f_{p \gamma} e^{-\left(E_{\gamma}^{\text {thin }} / E_{\gamma}\right)} E_{p}^{2} \frac{d N_{p}^{\text {iso }}}{d E_{p}}$, where the typical $\gamma$-ray energy is $E_{\gamma} \approx 0.1 E_{p}$.



FIG. 1: The interaction and attenuation lengths of highenergy photons and electron-positron pairs propagating in the Universe. An example of the source optical depth of photons in the case of bright LL GRB-like bursts is also shown for demonstration, where $r=10^{15} \mathrm{~cm}, \Gamma=10^{1.5}$, $L_{\gamma}^{b}=10^{48} \mathrm{erg} \mathrm{s}^{-1}, \alpha=1$ and $2.2, \varepsilon_{\mathrm{ob}}^{b}=10 \mathrm{keV}$ and $\varepsilon_{\mathrm{ob}}^{s a}=10^{0.5} \mathrm{eV}$.

Next, we briefly discuss the case of AGN flares. Following Ref. [11], let us adopt $L_{\gamma}^{b}=10^{45} \mathrm{erg} \mathrm{s}^{-1}$, $r=10^{16.5} \mathrm{~cm}$ and $\Gamma=10^{0.5}$ (corresponding to the duration of $\left.T \sim 10^{4-5} \mathrm{~s}\right)$. We can obtain $E_{\gamma}^{\text {thin }} \sim$ $10^{16.5} \mathrm{eV}\left(\tilde{\varepsilon}_{\mathrm{ob}}^{s a} / \tilde{\varepsilon}_{\mathrm{ob}}^{b}\right)^{\alpha-1} \Lambda$, taking $\varepsilon_{\mathrm{ob}}^{b} \sim 10 \mathrm{eV}$ and $L_{M} \sim$ $10^{47} \mathrm{erg} \mathrm{s}^{-1}$. Then, escape of UHE photons from the source is possible but it typically seems more difficult than the case of GRBs. The meson production efficiency is also estimated as $f_{p \gamma} \sim 0.1\left(E_{p} / E_{p}^{b}\right)^{\alpha-1}$, so that the expected fluence level of primary UHE photons can be similar to that shown in Fig. 2. Although detailed results depend on scenarios and parameters, the relevant processes are similar, and it is enough to show the case of GRBs for the demonstrative purpose of this work.

In order to prove acceleration of UHECRs, detections of particles with $E_{\nu} \approx 5 \mathrm{EeV} E_{p, 20}$ or $E_{\gamma} \approx 10 \mathrm{EeV} E_{p, 20}$ are favorable. However, very high-energy neutrino emission may be suppressed since charged mesons and muons can cool down before they decay [14]. It will be true especially in the case of GRB prompt emission since the comparison between $\pi^{ \pm}$'s lifetime and its synchrotron cooling time gives $E_{\nu}^{\mathrm{syn}} \approx 4.7 \times 10^{17} \mathrm{eV} \xi_{B}^{-1 / 2}\left(L_{\gamma, 48}^{b}\right)^{-1 / 2} \Gamma_{1.5}^{2} r_{14.5}$ above which the flux is suppressed by $\left(E_{\nu} / E_{\nu}^{\text {syn }}\right)^{-2}$.

Processes outside the source. - UHE photons, even if they can escape from the source, cannot avoid attenuation by the CIB, CMB, and CRB [1]. At the high energies of $\gtrsim 10 \mathrm{PeV}$, the attenuation lengths for pair creation and inverse-Compton scattering in the CMB are roughly $\lambda_{\gamma \gamma} \sim 2 \mathrm{Mpc} E_{\gamma, 18} / \ln \left(400 E_{\gamma, 18}\right)$ and $\lambda_{\mathrm{IC}} \sim$ $2 \mathrm{Mpc} \gamma_{e, 12} /\left[\ln \left(1800 \gamma_{e, 12}\right)-2\right]$, respectively, and numerically calculated lengths are shown in Fig. 1. The CRB has rather large uncertainty at present, so that we consider the extreme two cases: the non-CRB case and the case of the high CRB model developed in Ref. [21]. Secondary electron-positron pairs generated by pair creation are still energetic and upscatter cosmic background pho- 


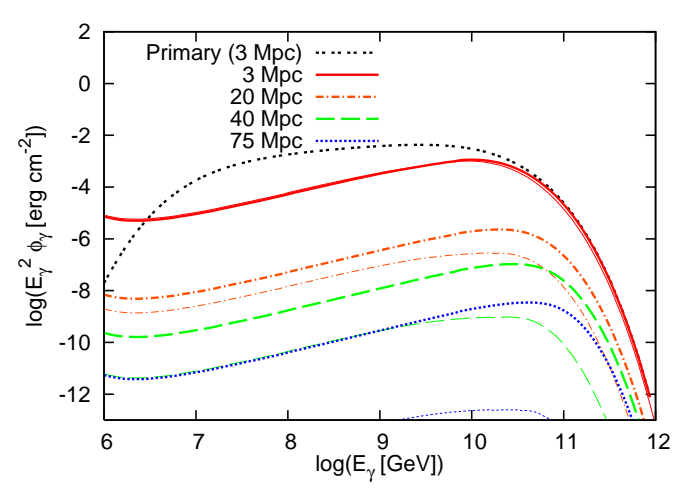

FIG. 2: Energy fluences of UHE photons from a LL GRB-like UHECR burst with $\tilde{\mathcal{E}}_{\mathrm{HECR}}^{\text {iso }}=10^{50.5} \mathrm{erg}$ for each distance. The primary $\gamma$-ray spectrum is also shown (see the caption of Fig. 1 for the source parameters). Thick lines show the non-CRB case while thin lines show the CRB case, with $B_{\mathrm{IG}}=10^{-13} \mathrm{G}$. The burst rates expected within each distance are $1 / 28000$, $1 / 94,1 / 12$, and $1 / 1.8 \mathrm{yr}^{-1}$.

tons. These boosted photons can create pairs as long as they are energetic, and the process repeats itself until the energy of degraded photons is in the $1-10 \mathrm{TeV}$ range. Hence, as a result of this cascade process, the effective attenuation lengths are longer than the original ones [1]. To take into account this cascade effect, we have solved cascade equations [1, 6], whose results agree with previous works [22, 23]. We can neglect double pair creation and Bethe-Heitler processes when $E_{\gamma}^{\max } \lesssim 10^{21} \mathrm{eV}[1]$.

In Fig. 2, the resulting UHE $\gamma$-ray spectra are demonstrated for the numerically calculated primary $\gamma$-ray spectrum. Cascaded $\gamma$ rays with $\lesssim 10^{19.5} \mathrm{eV}$ can enhance our chance to detect UHE signals from nearby transient sources (see below). For $D \sim 40 \mathrm{Mpc}$, the $\gamma$ ray fluence is $E_{\gamma}^{2} \phi_{\gamma} \sim 10^{-6.5} \mathrm{erg} \mathrm{cm}^{-2} f_{p \gamma,-1} \tilde{\mathcal{E}}_{\mathrm{HECR}, 50.5}^{\text {iso }}$ at $\sim 10^{19.5} \mathrm{eV}$ in the non-CRB case, allowing us to expect their detections if a UHECR burst occurs at $\sim 3 \mathrm{Mpc}$ (like Cen A), at $\sim 20 \mathrm{Mpc}$ (like the Virgo cluster), and at $\sim 40 \mathrm{Mpc}$ (like GRB 980425). For $D \sim 20 \mathrm{Mpc}$, we have $\mathcal{N} \sim 10$ events $f_{p \gamma,-1} \tilde{\mathcal{E}}_{\text {HECR }, 50.5}^{\text {iso }}$ by $\mathrm{PAO}\left(A \sim 3000 \mathrm{~km}^{2}\right)$. But, the results depend on the uncertain $\mathrm{CRB}$, which could make detections difficult for bursts at $\gtrsim 50 \mathrm{Mpc}$. They are also affected by the maximum UHECR energy.

The number of events $\mathcal{N}$ would not usually be large, so that space and time coincidence with lowenergy photons (e.g., $\mathrm{x} / \gamma$ rays) is important. Since the magnetic deflection angle is $\theta_{B} \approx \lambda_{\text {IC }}^{1 / 2} \lambda_{\text {coh }}^{1 / 2} / r_{L} \sim$ $2.6 \times 10^{-6} B_{\mathrm{IG},-13} \lambda_{\mathrm{coh}, \mathrm{kpc}}^{1 / 2} \gamma_{e, 13}^{-1 / 2} /\left[\ln \left(18000 \gamma_{e, 13}\right)-2\right]^{1 / 2}$, the magnetic time delay, which is typically the most important, is $\Delta t_{B} \approx \frac{1}{4} \frac{D}{c} \theta_{B}^{2} \sim$ 860 s $D_{40 \mathrm{Mpc}} B_{\mathrm{IG},-13}^{2} \lambda_{\mathrm{coh}, \mathrm{kpc}} \gamma_{e, 13}^{-1}$ [1, 23]. Hence, as long as the IGMF is weak enough, the magnetic time delay can be shorter than the burst duration of $T$ (e.g., $\sim 10^{2-3} \mathrm{~s}$ for GRBs), and coincident detections of

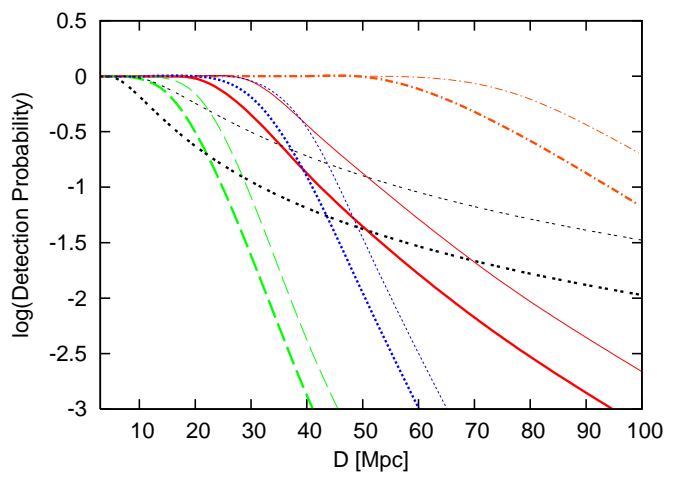

FIG. 3: The comparison of Poisson probabilities to detect UHE $(>10 \mathrm{EeV})$ photons and high-energy $(>10 \mathrm{PeV})$ neutrinos from a LL GRB-like UHECR burst. For UHE photons, $A=3000 \mathrm{~km}^{2}$ without the CRB (solid lines), $A=3000 \mathrm{~km}^{2}$ with the CRB (dashed lines), $A=3 \times 10^{5} \mathrm{~km}^{2}$ without the CRB (dotted-dashed lines), and $A=3 \times 10^{5} \mathrm{~km}^{2}$ with the CRB (dotted lines). For neutrinos, $A=1 \mathrm{~km}^{2}$ (double-dashed lines), assuming IceCube-like detectors. Thick and thin lines are for $\tilde{\mathcal{E}}_{\mathrm{HECR}}^{\text {iso }}=10^{50.5} \mathrm{erg}$ and $\tilde{\mathcal{E}}_{\mathrm{HECR}}^{\text {iso }}=10^{51} \mathrm{erg}$, respectively.

cascaded UHE photons can be expected. Note that such weak IGMFs are possible in voids, and the mean free path of UHE photons is $\gtrsim$ a few Mpc so that UHE photons may escape from the structured region (filaments and clusters) and UHE pairs may feel weak IGMFs only [8, 23]. On the other hand, UHECRs can have longer and sufficient time delays since they should feel stronger IGMFs in the structured region $(\sim \mathrm{nG}-\mu \mathrm{G})$ and the galactic magnetic field [13, 24]. If IGMFs are not weak or if there is the possible magnetic field of $\sim 0.1 \mu \mathrm{G}$ in the galactic halo, we expect coincidence only for noncascaded photons, and cascaded photons (especially for $\lesssim 10^{19.5} \mathrm{eV}$ photons) spread the signals out in time. The energy dependence is critical here, and lower-energy $\mathrm{GeV}-\mathrm{TeV}$ photons have the much longer duration 23.

In Fig. 3, we compare the Poisson probability $(\mathcal{P}=$ $\Sigma_{n} \mathcal{N}^{n} \mathrm{e}^{-\mathcal{N}} / n$ !) to detect $\geq 1$ events for neutrinos by $\mathrm{km}^{3}$ telescopes such as IceCube with that for UHE photons by large area detectors such as PAO and JEM-EUSO $(A \sim$ a few $\times 10^{5} \mathrm{~km}^{2}$ ) 25]. Spectra of both neutrinos and UHE photons are calculated for the same source parameters used in Figs. 1 and 2. UHE photons can be more useful to prove transient UHECR sources at from $\sim 10 \mathrm{Mpc}$ to $\sim 50-100 \mathrm{Mpc}$.

The burst rate of transient UHECR sources within 100 Mpc is estimated from $\rho$ as $\sim 1.3\left(\tilde{\mathcal{E}}_{\mathrm{HECR}, 50.5}^{\text {iso }}\right)^{-1} \mathrm{yr}^{-1}$ [13]. In fact, LL GRBs, hypernovae, and AGN flares may have corresponding rates of $\rho \sim 10^{2-3} \mathrm{Gpc}^{-3} \mathrm{yr}^{-1}$ [8, 11]. The expected rate is not so high, but there is still room to detect signals in the future.

Implications and discussions. - In this work, we have demonstrated that, for nearby sources within dozens of Mpc, detections of UHE photons by PAO and JEMEUSO can be expected and are important to identify the 
transient UHECR sources. They can also be useful to test the LIV which is often expected in quantum gravity theories [26]. Let us expand the energy-dependent light velocity as $c^{\prime}=c\left(E_{\gamma} / \zeta_{n} E_{\mathrm{pl}}\right)^{n}$, where $E_{\mathrm{pl}}$ is the Planck energy. Then, the LIV-induced time delay is written as $\Delta t_{\mathrm{LIV}} \simeq$ $(D / c)\left(E_{\gamma} / \zeta_{n} E_{\mathrm{pl}}\right)^{n}$. When UHE photons are coincident with low-energy photons during $T$, from $\Delta t_{\mathrm{LIV}}<T$, we obtain bounds of $\zeta_{1} \gtrsim 3.4 \times 10^{3} E_{\gamma, 19} T_{3}^{-1} D_{40 \mathrm{Mpc}}$ for $n=1$ and $\zeta_{2} \gtrsim 1.7 \times 10^{-3} E_{\gamma, 19} T_{3}^{-1 / 2} D_{40 \mathrm{Mpc}}^{1 / 2}$ for $n=2$. The current limits by Fermi observations of GRB 080916C are $\zeta_{1} \gtrsim 0.13$ and $\zeta_{2} \gtrsim 7.9 \times 10^{-10}$ [27]. Hence, possible detections of UHE photons may give us the most stringent limits on the LIV, as well as possible EeV neutrinos [20]. Even when observed UHE photons have time delays, we could potentially constrain the LIV since the energy dependence of $\Delta t_{\text {LIV }}$ is different from that of $\Delta t_{B}$. Furthermore, LIV-induced modifications to the attenuation may increase our chance to detect UHE photons [26].

UHE photons have two merits compared to neutrinos, in that (1) $\gtrsim \mathrm{EeV}$ neutrinos may be suppressed due to the meson cooling and their detections via Earth-skimming $\nu_{\tau}$ 's may not be so easy [25], and $(2) \sim \mathrm{PeV}$ neutrinos suitable for IceCube-like detectors directly suggest acceleration of $\sim 100 \mathrm{PeV}$ cosmic rays rather than UHECRs $\left(\gtrsim 10^{18.5} \mathrm{eV}\right)$. On the other hand, UHE $\gamma$-ray fluences depend on the CRB and the spectral shape at the highest energies. Another uncertainty comes from $f_{p \gamma}$ and $\tau_{\gamma \gamma}$ (e.g., in the mixed-composition scenario, it would be more difficult to detect neutrinos and photons [8]). Con- versely, detections of UHE photons will give us important information, e.g., enabling us to constrain the CRB.

The background UHE photons from the sources would not be so important compared to cosmogenic photons and current PAO limits [3], though the future anisotropy search via, e.g., finding multiplet events could be relevant especially if the duration of UHE photon emission is long. Their arrival distribution may also be expected to trace the matter distribution of the nearby Universe.

Finally, let us discuss associated $\mathrm{GeV}-\mathrm{TeV} \gamma$-ray signals. They should also be important since a significant fraction of UHE photons should be radiated as lowerenergy $\gamma$ rays via the synchrotron or inverse-Compton emission. But results would be rather sensitive to the IGMF in voids. If the IGMF is so weak, they may be detected as pair echoes, i.e., long lasting cascaded $\gamma$-ray emission [23]. If the IGMF is not weak, they may be detected as a pair halo [28] but their flux should be greatly reduced. If the IGMF is strong enough, UHE pairs will emit $\sim \mathrm{GeV} \gamma_{e, 13}^{2} B_{\mathrm{IG},-9}$ photons that could be detected by Fermi, though they are also be contaminated by accompanied leptonic components.

K.M. thanks C.D. Dermer, H. Takami, U. Jacob, T. Nakamura, S. Inoue, V.S. Berezinsky, and T. Ebisuzaki and especially appreciates comments by K. Ioka. He acknowledges support by a Grant-in-Aid from JSPS and by a Grant-in-Aid for the Global COE Program "The Next Generation of Physics, Spun from Universality and Emergence" from MEXT.
[1] P. Bhattacharjee and G. Sigl, Phys. Rep. 327, 109 (2000); C.D. Dermer, arXiv:0711.2804

[2] N. Nagano and A.A. Watson, Rev. Mod. Phys. 72, 689 (2000).

[3] J. Abraham et al., Science 318, 938 (2007); Astropart. Phys. 29, 188 (2008); Astropart. Phys. 29, 243 (2008).

[4] T. Kashti and E. Waxman, J. Cosmol. Astropart. Phys. 5 (2008) 006.

[5] J. Rachen and P.L. Biermann, Astron. Astrophys. 272, 161 (1993); C.A. Norman, D.B. Melrose, and A. Achterberg, Astrophys. J. 454, 60 (1995).

[6] C.D. Dermer et al., New J. Phys. 11, 065016 (2009).

[7] E. Waxman, Phys. Rev. Lett. 75, 386 (1995); M. Vietri, Astrophys. J. 453, 883 (1995).

[8] K. Murase, K. Ioka, S. Nagataki, and T. Nakamura, Astrophys. J. 651, L5 (2006); Phys. Rev. D 78, 023005 (2008).

[9] J. Arons, Astrophys. J. 589, 871 (2003).

[10] I. Zaw, G.R. Farrar, and J. Greene, Astrophys. J. 696, 1218 (2009).

[11] G.R. Farrar and A. Gruzinov, Astrophys. J. 693, 329 (2009).

[12] A. Atoyan and C.D. Dermer, Phys. Rev. Lett. 87, 221102 (2001); Astrophys. J. 586, 79 (2003).

[13] K. Murase and H. Takami, Astrophys. J. 690, L14 (2009).

[14] E. Waxman and J. Bahcall, Phys. Rev. Lett. 78, 2292 (1997); J.P. Rachen and P. Mészáros, Phys. Rev. D 58,
123005 (1998).

[15] K. Murase, Phys. Rev. D 76, 123001 (2007).

[16] P. Mészáros, Rep. Prog. Phys. 69, 2259 (2006).

[17] E.W. Liang et al., Astrophys. J. 662, 1111 (2007); D. Guetta and M.D. Valle, Astrophys. J. 657, L73 (2007).

[18] J. Granot et al., Astrophys. J. 677, 92 (2008).

[19] S. Razzaque, P. Mészáros, B. Zhang, Astrophys. J. 613, 1072 (2004).

[20] Z. Li and E. Waxman, arXiv:0711.4969.

[21] R.J. Protheroe and P. L. Biermann, Astropart. Phys. 6, 45 (1996).

[22] R.J. Protheroe, Mon. Not. R. Astron. Soc. 221, 769 (1986); S. Lee, Phys. Rev. D 58, 043004 (1998).

[23] K. Murase, K. Asano, and S. Nagataki, Astrophys. J. 671, 1886 (2007); K. Murase et al., Mon. Not. R. Astron. Soc. 396, 1825 (2009).

[24] S. Das, H. Kang, D. Ryu, and J. Cho, Astrophys. J. 682, 29 (2008).

[25] J. Ahrens et al., Astropart. Phys., 20, 507 (2004); G. Miele, S. Pastor, and O. Pisanti, Phys. Lett. B 634, 137 (2006); Y. Takahashi et al., New J. Phys. 11, 065009 (2009).

[26] G. Amelino-Camelia et al., Nature (London) 393, 763 (1998); U. Jacob and T. Piran, Nature Phys. 3, 87 (2007); Phys. Rev. D 78, 124010 (2008).

[27] A.A. Abdo et al., Science 323, 1688 (2009).

[28] F.A. Aharonian, P.S. Coppi, and H.J. Völk, Astrophys. J. 423, L5 (1994). 